\title{
Frequency-estimation-based signal-processing algorithm for white-light optical fiber Fabry-Perot interferometers
}

\author{
Fabin Shen and Anbo Wang
}

\begin{abstract}
A novel signal-processing algorithm based on frequency estimation of the spectrogram of single-mode optical fiber Fabry-Perot interferometric sensors under white-light illumination is described. The frequency-estimation approach is based on linear regression of the instantaneous phase of an analytical signal, which can be obtained by preprocessing the original spectrogram with a bandpass filter. This method can be used for a relatively large cavity length without the need for spectrogram normalization to the spectrum of the light source and can be extended directly to a multiplexed sensor system. Experimental results show that the method can yield both absolute measurement with high resolution and a large dynamic range. Performance analysis shows that the method is tolerant of background noise and variations of the source spectrum. (C) 2005 Optical Society of America
\end{abstract}

OCIS codes: $\quad 060.2370,120.3180,120.2650$.

\section{Introduction}

Fiber optic white-light interferometry has proved to be a practical method for measuring absolute optical path difference (OPD) with high accuracy and a large dynamic range. ${ }^{1-8}$ In a white-light measurement system, light from a low-coherence source, such as a light-emitting diode (LED), a superluminescent LED, or a broadband lamp, is transmitted to a single or multiplexed fiber sensors. A fringe pattern is produced by the light transmitted by or reflected from the sensor and recorded either spectrally by an optical spectrum analyzer (OSA) or temporally by a photodiode in a scanning interferometric system. ${ }^{1,2}$

When the interferogram of an interferometer is measured, the OPD of the interferometer can be estimated from the fringe patterns of the interference by fringe analysis. The fringe analysis approach most often used for white-light interferometers is the fringe peak tracking method, in which the peak position of a fringe or the fractional sample points between fringes in the interferogram are identified to determine the fringe order and to estimate the OPD

The authors are with the Center for Photonics Technology, Bradley Department of Electrical and Computer Engineering, Virginia Polytechnic Institute and State University, Blacksburg, Virginia 24061. F. Shen's e-mail address is fashen1@vt.edu.

Received 15 February 2005; revised manuscript received 7 April 2005; accepted 8 April 2005.

0003-6935/05/255206-09\$15.00/0

(C) 2005 Optical Society of America of the sensor. ${ }^{1-5}$ Although this fringe analysis method has been successfully used in many single interferometric sensor systems, it requires a high signal-tonoise ratio (SNR) to determine the fringe orders correctly and detect the peak positions accurately and cannot be directly applied to multiplexed sensor systems.

The most often used multiplexing scheme for fiber white-light interferometers is the path-matched differential interferometric coherence division multiplexing technique, in which the OPD of a receiving interferometer is scanned to match the OPDs of the interferometers interrogated. The multiplexed interferometers must have different OPDs that are larger than the coherence length of the light source. The path-matched differential interferometric fringe patterns from different sensors fall into different temporal ranges during the scanning of the receiving interferometer and can be separated in time or spatial domain. ${ }^{6-8}$

If a spectrogram of multiple interferometers is measured by an OSA, the fringe patterns from different interferometers will be mixed with one another. However, if the OPDs of the multiplexed interferometers are different, the measured spectrogram will have multiple frequency components that can be separated in the frequency domain.

In this paper we present a phase linear regressionbased frequency-estimation approach to the measurement of absolute OPDs of multiplexed Fabry-Perot (FP) interferometers from a measured spectrogram. We analyze the frequency components in a spectro- 


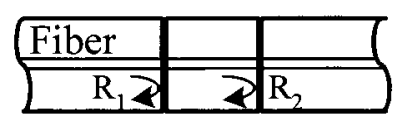

(a)

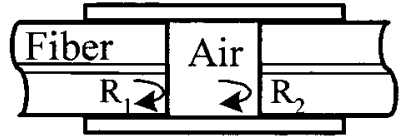

(b)
Fig. 1. FP interferometric sensors: (a) IFPI sensor, (b) EFPI sensor.

gram, use a finite impulse-response (FIR) filter to select the frequency component that corresponds to an individual FP interferometer, and estimate the frequency by linear regression of the instantaneous phase of the analytical signal. The OPD estimations are given for both known and unknown initial phases of FP interferometers.

The contents of this paper are arranged as follows: In Section 2, the frequency components of a spectrogram are analyzed, the analytical signal is introduced, and the frequency-estimation method based on instantaneous phase linear regression is presented. In Section 3, experimental results for multiplexed FP interferometers are demonstrated. The performance of the signal-processing method at several SNR levels is analyzed in Section 4, followed by a statement of our conclusions in Section 5 .

\section{Principles of Operation}

\section{A. Fabry-Perot Interferometers}

There are two main categories of fiber FP interferometric sensors, namely, intrinsic FP interferometers (IFPIs) and extrinsic FP interferometers (EFPIs). An IFPI sensor contains two internal partial reflection mirrors. The fiber between the reflectors serves as both a sensing element and a light waveguide. Lee and Taylor ${ }^{9,10}$ demonstrated some IFPI sensors by building dielectric mirrors into optical fiber, as shown in Fig. 1(a). An EFPI sensor contains a lead-in fiber with a partial mirror at the end face, a cavity of air or another transparent medium, and a reflector on the other end, which may also be a piece of fiber. Researchers at Virginia Tech developed some EFPI sensors constructed by forming an air gap between end faces of two uncoated fibers inserted into an alignment tube, as shown in Fig. 1(b). ${ }^{11,12}$ Sirkis et al. introduced in-line fiber etalons by fusion splicing two fibers with a section of hollow tube of the same outside diameter. ${ }^{13}$

The optical path length between the light reflected from the two partial mirrors in Figs. 1(a) and 1(b) is

$$
L=2 n_{e} d,
$$

where $n_{e}$ is the refractive index of the cavity medium and $d$ is the geometrical distance between the two reflectors.

When the light is launched into the FP interferometer, the light reflected from the two reflectors will interfere. Assuming that the reflection coefficients at the two reflectors, $R_{1}$ and $R_{2}$, are very small, the FP interferometer can be approximated to a two-beam or Fizeau interferometer with all the multipath reflections neglected. The electric field of the reflected light can be given as

$$
E=E_{1}+E_{2}=\eta_{1} R_{1} E_{0}+\eta_{2} R_{2} E_{0} \exp [j(k L+\phi)],
$$

where $E_{0}$ is the electric field of the incident light, $k$ is the wave number, $L$ is the OPD in Eq. (1), $\eta_{1}$ and $\eta_{2}$ are the coefficients of coupling efficiency of the light reflected into the lead-in fiber, and $\phi$ is a phase term that refers to reflection and light propagation. ${ }^{14}$

The intensity of the reflected light can be given as

$$
\begin{aligned}
I & =|E|^{2} \\
& =\left|E_{0}\right|^{2}\left[\eta_{1}{ }^{2} R_{1}{ }^{2}+\eta_{2}{ }^{2} R_{2}{ }^{2}+2 \eta_{1} \eta_{2} R_{1} R_{2} \cos (k L+\phi)\right] \\
& =I_{0}[A+B \cos (k L+\phi)],
\end{aligned}
$$

where $I_{0}$ is the intensity of the incident light at wave number $k$.

When light from a low-coherence light source is reflected by the FP cavity and measured by an OSA, the spectrogram of the interference can be given as

$$
I\left(k_{n}\right)=A I_{0}\left(k_{n}\right)+B I_{0}\left(k_{n}\right) \cos \left(k_{n} L+\phi\right)+v\left(k_{n}\right),
$$

where $I_{0}\left(k_{n}\right)$ are samples of the spectrum of incident light and $v\left(k_{n}\right)$ are the measurement noises. The coefficients $A, B, L$, and $\phi$ are assumed to be wavelength independent, which is true for most cases.

The measured spectrogram contains a background source spectrum $A I_{0}\left(k_{n}\right)$, an amplitude-modulated (AM) signal $B I_{0}\left(k_{n}\right) \cos \left(k_{n} L+\phi\right)$ with a carrier of frequency $L$ in the wave-number domain, and a noise term $v\left(k_{n}\right)$.

The goal of signal processing in white-light interferometry is to determine $L$ from the measured spectrum $I\left(k_{n}\right)$. This is equivalent to estimating carrier frequency $L$ from AM signal $B I_{0}\left(k_{n}\right) \cos \left(k_{n} L+\phi\right)$ corrupted by background noises $A I_{0}\left(k_{n}\right)$ and $v\left(k_{n}\right)$.

One may store a background spectrum of the light source $I_{0}\left(k_{n}\right)$ and obtain a normalized interference spectrogram:

$$
I_{N}\left(k_{n}\right)=\frac{I\left(k_{n}\right)}{I_{0}\left(k_{n}\right)}=A+B \cos \left(k_{n} L+\phi\right)+\xi\left(k_{n}\right) .
$$

Then the task becomes the classic problem of estimating the frequency of a sinusoid. However, unless the stored background source spectrum can be updated frequently, the normalization in Eq. (5) may give an error when the real light source spectrum, which is dependent on its driving current and ambient temperature, does not match the stored source spectrum.

A normalization method that can handle the variations of the light source spectrum was given in Refs. 5 and 6 . Two auxiliary spectral curves, $I_{\max }(k)$ and 

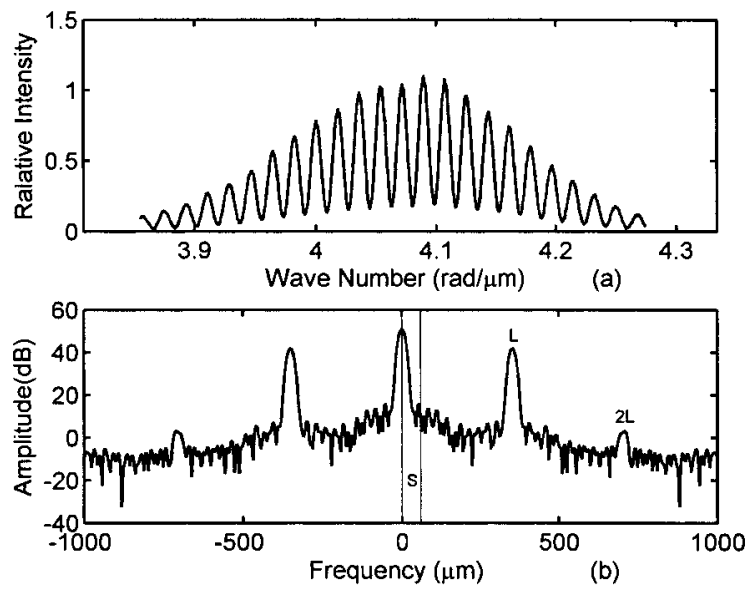

Fig. 2. (a) Spectrogram and (b) result of fast Fourier transformation of an EFPI sensor.

$I_{\text {min }}(k)$, which are the envelopes of the measured spectrogram, can be interpolated from the maxima and minima of the spectrogram and used to calculate the cosine term and eliminate the source spectrum in Eq. (4). However, this method also depends on the accuracy of extremum detection, which is difficult when there is a low SNR or when multiple sinusoids exist. It also requires that the number of extrema be large enough to yield a good interpolation, which means that the cavity length should be long enough.

We present a signal-processing approach that does not need normalization explicitly. The approach is based on frequency estimation of analytical signals, as discussed below.

\section{B. Analytical Signal}

The spectrogram of an EFPI cavity and its discrete Fourier-transformation result are shown in Fig. 2. The spectrogram has a variable of $k$ in radians per micrometer; its frequency component has units of micrometers.

The source spectrum, $I_{0}(k)$, falls into the lowfrequency region with spectral range $S$. The AM signal, $I_{0}(k) \cos (k L+\phi)$, shifts the spectrum of $I_{0}(k)$ to frequencies of $\pm L$. If $L$ is selected to be $L>2 S$ during the sensor fabrication, then the spectra of $I_{0}(k)$ and $I_{0}(k) \cos (k L+\phi)$ will not overlap, as Fig. 2 shows. We can use a bandpass filter to separate them to select the AM signal, $I_{0}(k) \cos (k L+\phi)$.

The analytical signal model of the AM signal can be expressed as

$$
I(k)=I_{0}(k) \exp [j(k L+\phi)] .
$$

We can use either of two methods to obtain this analytical signal from Eq. (4). One method is to design a single-band filter, which can be implemented by a double-band filter followed by a Hilbert transform, to select the single band of the AM signal, as shown in

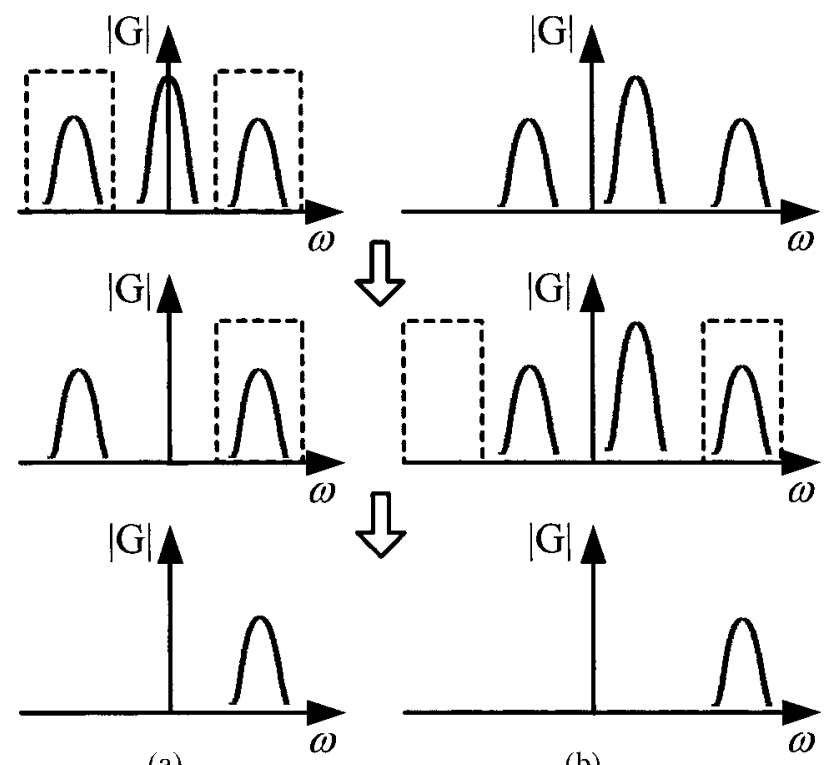

(a)

(b)

Fig. 3. Methods for obtaining analytical signals: (a) single-band filter, (b) frequency shift.

Fig. 3(a). The filtering and Hilbert transformation can be calculated efficiently by fast Fourier transformation. The other method is first to shift the frequency components by multiplying Eq. (4) by $\exp \left(j k L_{0}\right)$ and filtering the signal with a double-band filter. Then an analytical signal with central frequency $L+L_{0}$ can be obtained, as shown in Fig. 3(b). Schematics of these two methods are shown in Fig. 3.

We use a digital FIR bandpass filter whose passband center frequency is close to $L$ to select the AM signal in Eq. (4). It is well known that a FIR filter is a linear phase filter. It introduces a pure delay into the signal without giving any other distortion to the signal in the passband. The pure delay of samples caused by the FIR filter is

$$
n_{d}=\left(M_{f}-1\right) / 2 \text {, }
$$

where $M_{f}$ is the order of the FIR filter. One can simply shift the filtered signal backward by $n_{d}$ samples to compensate for the phase change caused by the FIR filter.

If the wave number is uniformly spaced in steps of $k_{s}$ from starting wave number $k_{0}$, then, after filtering and backward shifting, the analytical signal can be written as

$$
\begin{aligned}
\bar{x}_{n} & =\bar{A}_{n} \exp \left[j\left(k_{n} L+\phi\right)\right]+\bar{v}_{n} \\
& =\bar{A}_{n} \exp \left[j\left(k_{s} L n+k_{0} L+\phi\right)\right]+\bar{v}_{n} \\
(n & =1,2, \ldots N),
\end{aligned}
$$

where $\bar{A}_{n}$ is the amplitude after filtering, $k_{0}$ is the starting wave number, $k_{s}$ is the wave-number step, and $\bar{v}_{n}$ is the residual noise after filtering. Carrier 
frequency $L$ or normalized angular carrier frequency

$$
\omega=k_{s} L
$$

can be estimated by the method of phase linear regression discussed below.

\section{Frequency Estimation}

A frequency estimation based on linear regression of the instantaneous signal phase was given in Ref. 15 . The frequency and phase estimation of sinusoids from a sequence of signal samples is given as

$$
x_{n}=A \exp [j(\omega n+\phi)]+v_{n},
$$

where $A, \omega$, and $\phi$ are unknown parameters to be estimated and $v_{n}$ is additive Gaussian white noise.

The difference between Eqs. (8) and (10) is that amplitude $\bar{A}_{n}$ in Eq. (8) is wave-number dependent, whereas amplitude $A$ in Eq. (10) is constant. However, the derivations of the unwrapped phases of Eqs. (8) and (10) are the same as shown in Eq. (11). Thus the frequency-estimation method in Ref. 15 can be extended to the AM analytical signal model.

The unwrapped phase of the sinusoids in Eq. (8) can be given as

$$
\phi_{n}=\left[\angle \bar{x}_{n}\right]_{2 \pi}=k_{n} L+\phi+\zeta_{n} \quad(n=[1, \ldots, N]),
$$

where $\left[\angle \bar{x}_{n}\right]_{2 \pi}$ means $\tan ^{-1}\left[\operatorname{Im}\left(\bar{x}_{n}\right) / \operatorname{Re}\left(\bar{x}_{n}\right)\right]+2 m \pi, \zeta_{n}$ is the equivalent phase noise of additive noise $\bar{v}_{n}$ in Eq. (8), and $N$ is the length of the unwrapped phase sequence.

The parameters $L$ and $\phi$ can be estimated by the method of least-squares estimation or linear regression to minimize the square error:

$$
S=\Sigma\left[\phi_{n}-k_{n} \hat{L}-\hat{\phi}\right]^{2} .
$$

The solution is

$$
\left[\begin{array}{l}
\hat{L} \\
\hat{\phi}
\end{array}\right]=\left(A^{T} A\right)^{-1} A^{T} \Phi,
$$

where

$$
\begin{aligned}
A & =\left[\begin{array}{cccc}
k_{1} & k_{2} & \ldots & k_{N} \\
1 & 1 & \ldots & 1
\end{array}\right]^{T}, \\
\Phi & =\left[\begin{array}{llll}
\phi_{1} & \phi_{2} & \ldots & \phi_{N}
\end{array}\right]^{T} .
\end{aligned}
$$

Estimation $\hat{L}$ usually does not have high precision, especially when the SNR is not high. A simulation result of the variance of the normalized frequency estimation for unknown frequency and phase is given in Subsection 4.A below. In practice, phase term $\phi$ in Eqs. (4) and (8), which is a constant, can be known $a$ priori by either accurate modeling or calibration in advance. We can use it to get a higher frequencyestimation precision than that of an unknown phase. One can obtain averages of multiple estimations $\hat{L}$ and $\hat{\phi}$ from Eq. (13) and store them as calibrated values of $\bar{L}$ and $\bar{\phi}$. The modulus of $\bar{\phi}$ with $2 \pi, \tilde{\phi}$ $=[\bar{\phi}]_{2 \pi}$, whose value falls within $[-\pi, \pi]$, can be used as a good calibrated approximation of $\phi$.

If the estimation error $\Delta L=\hat{L}-L$ is less than $\lambda / 2$, where $\lambda$ is the wavelength of light, the phase error caused by $\Delta L$,

$$
\Delta \phi_{n}=k_{n} \Delta L,
$$

will be in the range $[-\pi, \pi]$. Therefore, for the estimation of $\hat{L}$ and $\hat{\phi}$ from Eq. (13), we can select

$$
\Delta \phi=[\hat{\phi}-\phi]
$$

or

$$
[\hat{\phi}-\tilde{\phi}],
$$

whose value falls within $[-\pi, \pi]$, as an estimation of phase error caused by $\Delta L$. Then we can estimate $\Delta L$ by minimizing the variance

$$
S=\Sigma\left[\Delta \phi-k_{n} \Delta \hat{L}\right]^{2}
$$

with the solution

$$
\Delta \hat{L}=\left(C^{T} C\right)^{-1} C^{T} D
$$

where

$$
\begin{aligned}
& C=\left[\begin{array}{llll}
k_{1} & k_{2} & \ldots & k_{N}
\end{array}\right]^{T}, \\
& D=\left[\begin{array}{llll}
\Delta \phi & \Delta \phi & \ldots & \Delta \phi
\end{array}\right]^{T} .
\end{aligned}
$$

We can use the estimation of Eq. (17) as compensation for the frequency estimation of Eq. (13). The compensated frequency estimation for known $\phi$ is

$$
\tilde{L}=\hat{L}+\Delta \hat{L} \text {. }
$$

It will have higher precision than that of the unknown $\phi$. The simulation results of frequency estimation for unknown and known phases, $\hat{L}$ in Eq. (13) and $\tilde{L}$ in Eq. (18), at different SNR levels, are shown in Subsection 4.A below.

It needs to be noted that the signal-processing approach can be extended to a multiplexing system in which the measured spectrogram has multiple frequency components that correspond to different interferometers. The spectrogram of multiplexed interferometers can be given as 


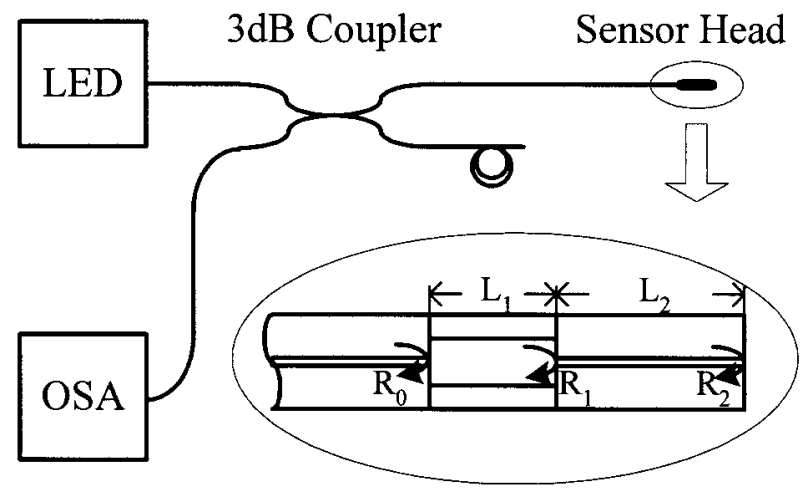

Fig. 4. Schematic of the experimental setup.

$$
I\left(k_{n}\right)=A I_{0}\left(k_{n}\right)+\sum_{i=1}^{M} B_{i} I_{0}\left(k_{n}\right) \cos \left(2 \pi k_{n} L_{i}+\phi_{i}\right)+v\left(k_{n}\right),
$$

where $M$ is the number of frequency components. We can use bandpass filters with different central frequencies to select and estimate each frequency component separately.

The signal-processing method for multiple frequency components with known phases is summarized as follows:

(a) If needed, manipulate the measured spectrogram to be equally spaced in wave numbers.

(b) Use a FIR bandpass filter to select a single frequency component and obtain a single-band analytical signal as described in Eq. (8).

(c) Obtain the unwrapped phase of the analytical signal.

(d) Obtain an estimation of $\hat{L}$ and $\hat{\phi}$ from Eq. (13).

(e) Obtain the phase difference between $\hat{\phi}$ and the known phase from Eq. (15).

(f) Obtain a compensated frequency estimation from Eqs. (17) and (18).

(g) Repeat steps (b)-(f) until all frequencies are estimated.

The experimental results of this method for multiplexed FP interferometers are demonstrated in Section 3 below.

\section{Experimental Results}

We use a simple setup to evaluate the signalprocessing algorithm for multiplexed FP interferometers. The setup of the experiment is shown schematically in Fig. 4.

We construct the sensor head by splicing a piece of hollow tube between single-mode fibers as described in Ref. 13. The interfaces between the silica fibers and the air in the hollow tube serve as partial reflection mirrors, which give reflections $R_{0}$ and $R_{1}$. The cleaved end face of the single-mode fiber serves as another mirror that gives reflection $R_{2}$. Each pair of these reflections will interfere and construct a FP interferometer. Thus the sensor head contains an EFPI air gap cavity formed by $R_{0}$ and $R_{1}$, an IFPI silica fiber cavity by $R_{1}$ and $R_{2}$, and an extra FP cavity by $R_{0}$ and $R_{2}$.

Light from a low-coherence light source is launched to the sensor head through a single-mode fiber and a $3 \mathrm{~dB}$ directional coupler. The LED has a central wavelength of $1.55 \mu \mathrm{m}$ and a FWHM bandwidth of $80 \mathrm{~nm}$. The light reflected from the sensor head is coupled to an OSA (Ando AQ-6315A) by the coupler, while we eliminate the reflection from the other arm by curling the fiber into several turns of small diameter. The OSA has a wavelength accuracy of $\pm 0.05 \mathrm{~nm}$.

The electric fields of these reflections coupled back to the lead-in fiber can be given as

$$
\begin{aligned}
E= & E_{1}+E_{2}+E_{3} \\
= & \eta_{0} R_{0} E_{0}+\eta_{1} R_{1} E_{0} \exp \left[j\left(k L_{1}+\phi_{1}\right)\right] \\
& +\eta_{2} R_{2} E_{0} \exp \left[j\left(k L_{1}+\phi_{1}\right)\right] \exp \left[j\left(k L_{2}+\phi_{2}\right)\right],
\end{aligned}
$$

where $\eta_{1}, \eta_{2}$, and $\eta_{3}$ are the coupling coefficients of $R_{1}$, $R_{2}$, and $R_{3}$ into the lead-in fiber and $L_{1}$ and $L_{2}$ are the OPDs of the air gap and the silica fiber FP cavities, respectively. All the multipath reflections are neglected owing to the low reflections $R_{0}, R_{1}$, and $R_{2}$.

The intensity of these reflections at wave number $k$ can be given as

$$
\begin{aligned}
I= & |E|^{2} \\
= & I_{0}\left[A+B \cos \left(k L_{1}+\phi_{1}\right)+C \cos \left(k L_{2}+\phi_{2}\right)\right. \\
& \left.+D \cos \left(k L_{3}+\phi_{3}\right)\right],
\end{aligned}
$$

where

$$
\begin{aligned}
& I_{0}=\left|E_{0}\right|^{2}, \\
& A=\eta_{0}^{2} R_{0}^{2}+\eta_{1}^{2} R_{1}^{2}+\eta_{2}^{2} R_{2}^{2}, \\
& B=2 \eta_{0} \eta_{1} R_{0} R_{1}, \\
& C=2 \eta_{1} \eta_{2} R_{1} R_{2}, \\
& D=2 \eta_{0} \eta_{2} R_{0} R_{2}, \\
& L_{3}=L_{1}+L_{2}, \\
& \phi_{3}=\phi_{1}+\phi_{2} .
\end{aligned}
$$

The measured spectrogram of the sensors can be expressed as Eq. (19) with three sinusoidal components corresponding to the three FP cavities. A measured spectrogram and its discrete Fourier transformation result are shown in Figs. 5(a) and 5 (b), respectively. The OPDs of the air gap FP cavity and the glass fiber FP cavity, $L_{1}$ and $L_{2}$, respectively, were selected to be 384 and $1315 \mu \mathrm{m}$. The frequencies of the sinusoids corresponding to $L_{1}$ and $L_{2}$ were located separately in the frequency domain. 

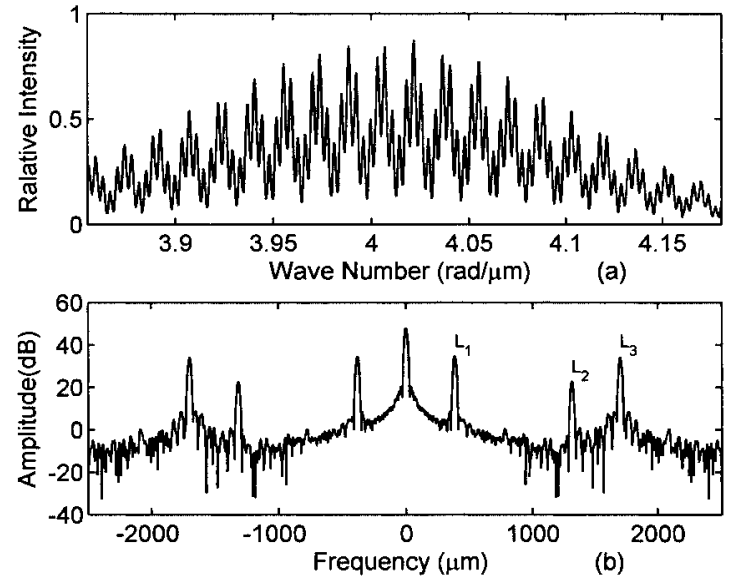

Fig. 5. (a) Spectrogram and (b) result of fast Fourier transformation of multiplexed FP sensors.

We investigated the temperature dependence of these two FP cavities to verify the performance of the signal-processing algorithm discussed in Section 2. We estimated $L_{1}$ and $L_{2}$ separately, following steps (b)-(f). Two Hanning-windowed FIR bandpass filters were used to select the frequency components corresponding to $L_{1}$ and $L_{2}$.

When the sensor head is put into a temperaturevarying environment, the OPDs of the FP interferometers will change owing to thermal expansions of the cavity lengths and thermo-optic effects of the media between the reflectors. The OPD change of a FP interferometer can be given as

$$
\Delta L=2\left(\Delta n_{e} d+n_{e} \Delta d\right)=L\left(\frac{\Delta n_{e}}{n_{e}}+\frac{\Delta d}{d}\right) .
$$

For the air gap FP cavity, one can assume that the refractive index of air is not temperature dependent. The OPD change of $L_{1}$ is due only to the thermal expansion of the silica hollow tube:

$$
\Delta L_{1}=L_{1} \frac{\Delta d}{d}=L_{1} \alpha_{T} \Delta T
$$

where $\alpha_{T}$ is the coefficient of thermal expansion of the silica hollow tube and $\Delta T$ is the temperature change. For silica, $\alpha_{T} \approx 0.5 \times 10^{-6}\left(1 /{ }^{\circ} \mathrm{C}\right)$.

For the silica fiber FP cavity, the OPD change is due to both the thermo-optic effect and the thermal expansion of the silica fiber. The refractive-index change of the fiber caused by a temperature change is

$$
\Delta n_{e}=\frac{\partial n_{e}}{\partial T} \Delta T
$$

where $\partial n_{e} / \partial T$ is a thermo-optic coefficient, which is $\sim 1.0 \times 10^{-5} /{ }^{\circ} \mathrm{C}$ for silica fiber. Thus the total OPD change $\Delta L_{2}$ can be written as
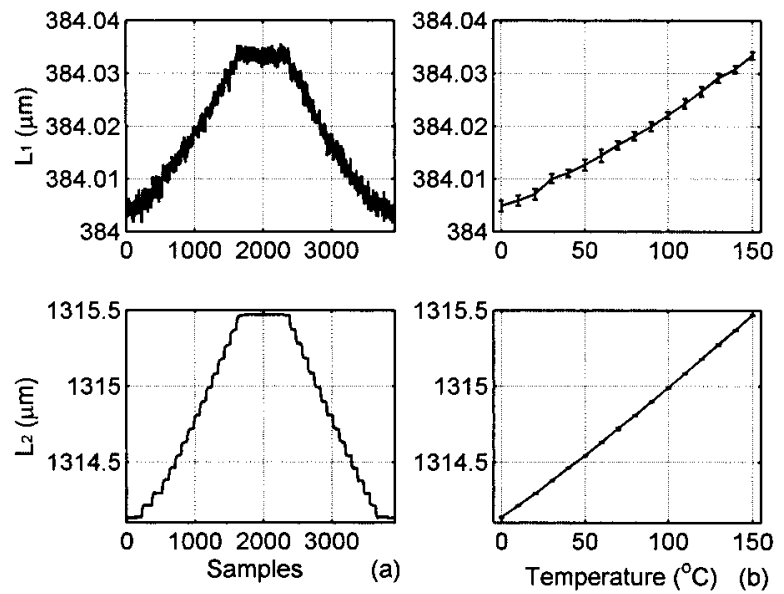

Fig. 6. Temperature responses of the multiplexed FP sensors: (a) OPDs during the temperature cycle, (b) OPDs versus temperature.

$$
\Delta L_{2}=L_{2}\left(\frac{\partial n_{e}}{\partial T} / n_{e}+\alpha_{T}\right) \Delta T=L_{2}\left(\sigma_{T}+\alpha_{T}\right) \Delta T
$$

where $\sigma_{T}$ and $\alpha_{T}$ are effective thermo-optic and thermal expansion coefficients of silica fiber, respectively. $\sigma_{T}$ is much larger than $\alpha_{T}$, which means that the thermo-optic effect is dominant for the temperature dependence of the OPD of the silica fiber cavity.

The sensor head was tested in a temperature chamber with a temperature range of $0^{\circ}-150{ }^{\circ} \mathrm{C}$ and temperature steps of $10{ }^{\circ} \mathrm{C}$. The time at each step during the increasing-decreasing cycle was $1 \mathrm{~h}$, except at $150{ }^{\circ} \mathrm{C}$, where it was $8 \mathrm{~h}$. The history curves and temperature dependences of $L_{1}$ and $L_{2}$ are shown in Figs. 6(a) and 6(b), respectively.

Figure 6(a) shows the measured OPDs of the two FP cavities during the temperature cycle. Figure 6(b) shows the temperature dependence of the OPDs. The standard deviations of the estimations of $L_{1}$ and $L_{2}$ at $150{ }^{\circ} \mathrm{C}$ are 0.8 and $3.0 \mathrm{~nm}$, respectively. The uncertainty of the OPD measurement, $\delta L / L$, is $\sim 2.2 \times 10^{-6}$ in both cases.

Experimental results show that the method can attain both absolute measurement of OPDs with high resolution and a large dynamic measurement range. For example, the estimation of the OPD of the silica fiber FP cavity has a $3.0 \mathrm{~nm}$ resolution in a dynamic range of $14 \mu \mathrm{m}$.

\section{Performance Analysis}

\section{A. Background Noise}

The background noises include $A I_{0}\left(k_{n}\right)$, which is a slowly varying process from the light source, and $v\left(k_{n}\right)$, which can be modeled as a Gaussian random process. $v\left(k_{n}\right)$ may include the detector's dark-current noise, the shot noise, and the thermal noise from the electric circuits.

The frequency estimation based on the phase linear regression in Eq. (13) is the optimal maximumlikelihood estimation for Eq. (11) when $\zeta_{n}$ is Gaussian 


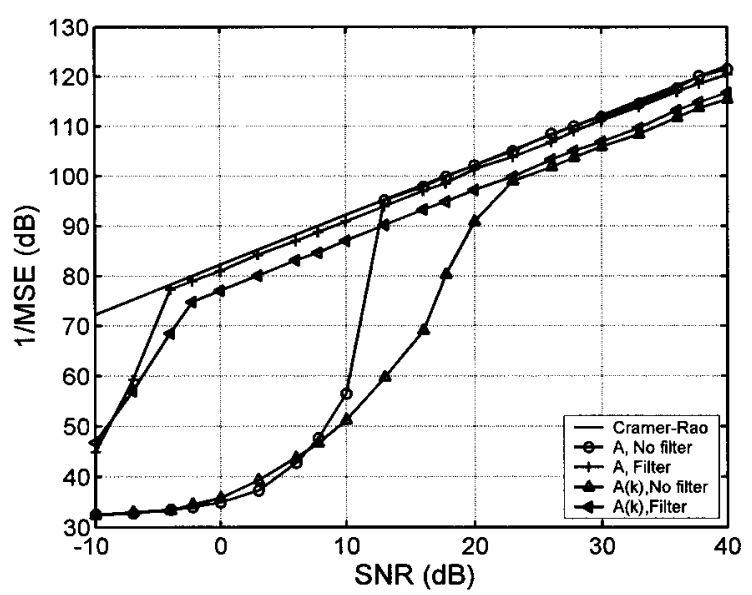

Fig. 7. Simulation results of frequency estimations for unknown phases.

white noise. ${ }^{16,17}$ When the SNR is moderately high, the estimator can attain the Cramer-Rao bound for a sinusoidal signal with constant amplitude, as described in Eq. (10). ${ }^{15,16}$ However, when the sinusoidal signal is amplitude modulated as described in Eq. (8), the average SNR is decreased; thus the estimator will have a larger estimation error for the AM signal than for that of pure sinusoids. It can also be expected that prefiltering with a bandpass filter will reject the noise outside the passband and increase the SNR greatly. Therefore prefiltering will greatly improve the performance of the estimator, especially for a low-SNR case.

The performance of the estimator [Eq. (13)] for an unknown phase was investigated for AM and pure sinusoidal signals at different SNR levels. The simulation results with and without prefiltering were compared. The simulation was based on a sequence of 1000 samples of a sinusoid whose normalized frequency is 0.024 . Figure 7 shows the relationship between the mean-square errors (MSEs) of the frequency estimation and SNR levels. It can be seen from Fig. 7 that estimator (13) can yield a good estimation for a high SNR but poor performance for a low SNR. Prefiltering will greatly improve performance at low SNR levels. The Cramer-Rao bound of the frequency estimation for Eq. (10) with an unknown phase ${ }^{15,17}$ is also plotted for comparison.

Frequency estimations for unknown phase and known phase, as described in Eqs. (13) and (18), are compared in Fig. 8. The simulation data were generated for a FP interferometer with an OPD of $360 \mu \mathrm{m}$ illuminated by a LED with a Gaussian shaped spectrum whose FWHM bandwidth is $80 \mathrm{~nm}$. It can be seen that the frequency-estimation error for a known phase can be much smaller than that of an unknown phase, which means that the frequency estimation in Eq. (18) can have higher precision than that of Eq. (13).

The frequency estimation for a known phase given by Eqs. (13), (17), and (18) is based on the assumption that the phase error caused by the frequency-estimation error is within $[-\pi, \pi]$. However, when the SNR is low, the frequency-

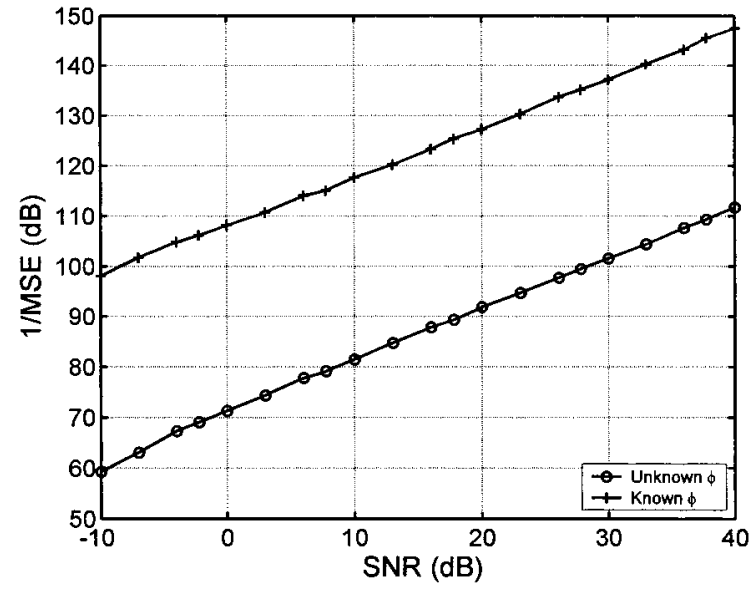

Fig. 8. Comparison of frequency-estimation errors for unknown and known phases.

estimation error given by Eq. (13) may be large; thus the phase error selected by Eq. (15) may have a $2 \pi$ ambiguity, which will lead to error jumping of the frequency estimation. This $2 \pi$ ambiguity is similar to that of the order-determination ambiguity problem in the fringe peak tracking-based signalprocessing approach. For example, to ensure a coarse estimation error $\Delta L=\hat{L}-L$ of less than $\lambda / 2$, from Eq. (5), for a wave-number step of $k_{s}=4.2$ $\times 10^{-4} \mathrm{rad} / \mu \mathrm{m}$, the frequency-estimation error should be less than $3.2 \times 10^{-4}$, which requires that $1 / \mathrm{MSE}$ in Fig. 7 be larger than $70 \mathrm{~dB}$. Thus it can be seen that the required SNR should be larger than $0 \mathrm{~dB}$ to yield a reasonable confidence interval to prevent a $2 \pi$ ambiguity problem.

\section{B. Amplitude Noise}

The frequency range of $I_{0}(k)$ is $S$, as Fig. 2 shows. We consider only the amplitude noise whose bandwidth is limited by $S$. In practice, the amplitude variation caused by the driving current and by the change in ambient temperature of the LED is usually of low frequency. The variation of the amplitude can thus be eliminated from Eq. (11) because only the phase of the analytical signal is obtained.

Figure 9 shows the experimental results of the EFPI cavity shown in Fig. 2 illuminated by (A) a LED of total power $50 \mu \mathrm{W}$, (B) the same LED, whose power of $60 \mu \mathrm{W}$ has been increased by an increase in driving current, and (C) a superluminescent LED of total power of $1 \mathrm{~mW}$. The source spectra of several illuminations are shown in Fig. 9(a); the OPD estimations are shown in Fig. 9(b). Although the source spectra are different, which means that the amplitudes of the analytical signal vary greatly, the OPD readings are consistent with a small variation of $2 \mathrm{~nm}$.

\section{Phase Noise}

The estimation [Eq. (13)] for the model in Eq. (11) is optimal when $\zeta_{n}$ is Gaussian white noise. However, 

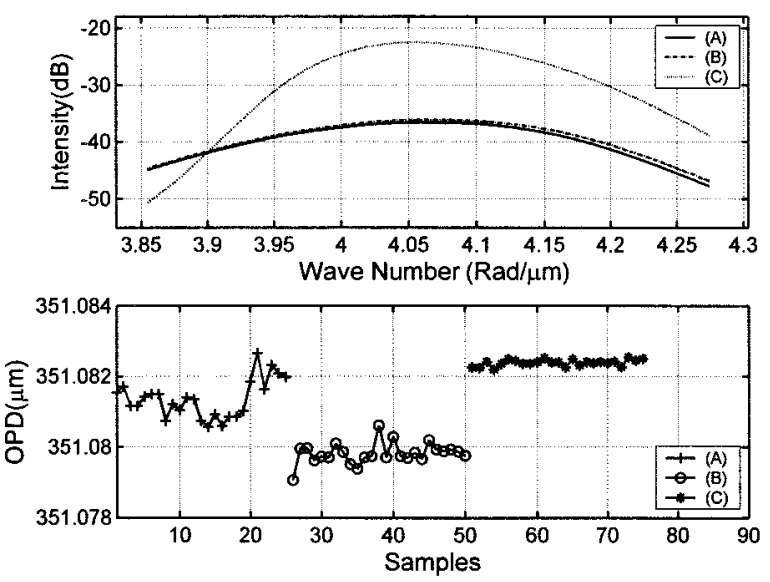

Fig. 9. OPD estimations under three illuminations: (a) source spectra, (b) OPD estimations.

phase noise $\zeta_{n}$ in a FP interferometer, which is wave number and geometric length dependent, is not really white. The dependency of phase noise can be given as

$$
\delta \phi=\delta k L+k \delta L=\delta k L+k \alpha L=(\delta k+k \alpha) L,
$$

where $\alpha$ is the ratio of change in the OPD. Wavenumber error $\delta k$ is caused by jittering of the light source and by the wavelength measurement uncertainty of the OSA. OPD error $\delta L$ is caused by the variation in the OPD during measurement of the spectrogram. Usually the wavelength uncertainty of an OSA is the main source of phase noise.

From Eq. (26) it is evident that phase noise $\delta \phi$ caused by $\delta k$ and $\delta L$ is proportional to $L$. Therefore the SNR level for large $L$ is reduced because of the larger phase noise. This is the reason why the frequency-estimation precision for a small $L$ is higher, as the experimental result in Section 3 shows. Another disadvantage of a large $L$ is that it lowers the interference contrast, which decreases the signal power and the SNR. There is also a higher probability of occurrence of $2 \pi$ ambiguity for large $L$ because a larger frequency-estimation error $\Delta L$ may occur. Therefore, if possible, one should select a shorter $L$ that satisfies the condition $L>2 S$, as discussed in Subsection 2.B, for both precision of estimation and avoidance of ambiguity.

\section{Two-Beam Approximation}

We use a two-beam approximation model [Eq. (3)] to describe a FP interferometer. Multipath reflections are neglected because they are low reflections. However, multipath reflections will give some harmonic frequency components to the measured spectrogram. The side peaks of the harmonics can be observed in Fig. 2.

Because a bandpass filter is applied to select the fundamental frequency components, the influence of these harmonics has been reduced to a minimum. If the frequency component of another interferometer coincides with the positions of these harmonics in a multiplexed sensor system, cross talk will occur. Thus, one should carefully design the OPDs of the sensors and locate the frequencies properly to prevent cross talk between interferometers. However, the power of the harmonics is rather low compared with the fundamental frequency component, $-28 \mathrm{~dB}$ for a $4 \%$ air-glass interface reflection, so the influence of cross talk can be reasonably neglected.

\section{Conclusions}

A frequency-estimation-based signal-processing algorithm for white-light optical fiber Fabry-Perot interferometers has been presented. The frequency estimation is based on linear regression of the instantaneous phase of the analytical signal, which can be obtained from the spectrogram of a FP interferometer. Experimental results for multiplexed FP interferometers show that the method can achieve both absolute optical path difference estimation with high resolution and a large dynamic range. We have analyzed the performance of the algorithm by considering various noises at different levels.

This research was supported in part by U.S. Department of Energy under grant DE-FC3601G011050 and U.S. National Science Foundation under grant CMS-0427951.

\section{References}

1. B. T. Meggit, "Fiber optic white light interferometric sensors," in Optical Fiber Sensor Technology, K. T. V. Grattan and B. T. Meggitt, eds. (Kluwer Academic, 2000), Vol. 4, 193-238.

2. S. Chen, A. W. Palmer, K. T. V. Grattan, and B. T. Meggitt, "Digital signal-processing techniques for electronically scanned optical-fiber white-light interferometry," Appl. Opt. 31, 6003-6010 (1992).

3. B. Qi, G. R. Pickrell, J. Xu, P. Zhang, Y. Duan, W. Peng, Z. Huang, W. Huo, H. Xiao, R. G. May, and A. Wang, "Novel data processing techniques for dispersive white light interferometer," Opt. Eng. 42, 3165 (2003).

4. R. Cortés, A. V. Khomenko, A. N. Starodumov, N. Arzate, and L. A. Zenteno, "Interferometric fiber-optic temperature sensor with spiral polarization couplers," Opt. Commun. 15, 268-272 (1998).

5. J. Tapia-Mercado, A. V. Khomenko, and A. Garcia-Weidner, "Precision and sensitivity optimization for white-light inteferometric fiber-optic sensors," J. Lightwave Technol. 19, 70-74 (2001).

6. J. L. Brooks, R. H. Wentworth, R. C. Youngquist, M. Tur, B. Y. Kim, and H. J. Shaw, "Coherence multiplexing of fiber-optic interferometric sensors," J. Lightwave Technol. 3, 1062-1072 (1985).

7. W. V. Sorin and D. M. Baney, "Multiplexed sensing using optical low-coherence reflectometry," IEEE Photon. Technol. Lett. 7, 917-919 (1995).

8. Y.-L. Lo, "Study of cross-talk of parallel Fabry Perot sensors in path-matching differential interferometry," Opt. Lasers Eng. 31, 401-410 (1999).

9. C. E. Lee and H. F. Taylor, "Interferometric optical fiber sensors using internal mirrors," Electron Lett. 24, 193-194 (1988).

10. C. E. Lee and H. F. Taylor, "In-line Fiber Fabry Perot interferometer with high reflectance internal mirrors," J. Lightwave Technol. 10, 1376-1379 (1992).

11. K. A. Murphy, M. F. Gunther, A. Wang, R. O. Claus, and A. M. Vengsarkar, "Extrinsic Fabry Perot optical fiber sensor," in 
Eighth Optical Fiber Sensors Conference (Institute of Electrical and Electronics Engineers, 1992), pp. 193-196.

12. A. Wang, H. Xiao, J. Wang, Z. Wang, W. Zhao, and R. G. May, "Self-calibrated interferometric-intensity-based optical fiber sensors," J. Lightwave Technol. 19, 1495-1501 (2001).

13. J. Sirkis, T. A. Berkoff, R. T. Jones, H. Singh, A. D. Kersey, E. J. Friebele, and M. A. Putnam, "In-line fiber etalon (ILFE) fiber-optic strain sensors," J. Lightwave Technol. 13, 12561268 (1995).

14. M. Han, Y. Zhang, F. Shen, G. R. Pickrell, and A. Wang, "Signal-processing algorithm for white-light optical fiber ex- trinsic Fabry-Perot interferometric sensors," Opt. Lett. 29, 1736-1378 (2004).

15. S. A. Tretter, "Estimating the frequency of a noisy sinusoid by linear regression," IEEE Trans. Inf. Theory IT-31, 832-835 (1985).

16. S. M. Kay, "A fast and accurate single frequency estimator," IEEE Trans. Acoust. Speech Signal Process. 37, 1987-1990 (1989).

17. D. C. Rife and R. R. Boorstyn, "Single-tone parameter estimation from discrete-time observations," IEEE Trans. Inf. Theory IT-20, 591-598 (1974). 